\title{
Six-month atrophy in MTL structures is associated with subsequent memory decline in elderly controls
}

\author{
E.A. Murphy, B.A. ${ }^{a}$, D. Holland, Ph.D. a,c, M. Donohue, Ph.D.d, L.K. McEvoy, Ph.D. b,c , D.J. \\ Hagler Jr., Ph.D.b,c, A.M. Dale, Ph.D. a,b,c, J. B. Brewer, M.D., Ph.D. a,b, ${ }^{*}$, and the Alzheimer's \\ Disease Neuroimaging Initiative \\ aDepartment of Neurosciences at the University of California, San Diego, CA, USA \\ bepartment of Radiology, at the University of California, San Diego, CA, USA \\ 'Multimodal Imaging Laboratory, at the University of California, San Diego, CA, USA \\ ${ }^{\mathrm{d} D i v i s i o n}$ of Biostatistics \& Bioinformatics at the University of California, San Diego, CA, USA
}

\begin{abstract}
Neurodegeneration precedes the onset of dementias such as Alzheimer's by several years. Recent advances in volumetric imaging allow quantification of subtle neuroanatomical change over time periods as short as six months. This study investigates whether neuroanatomical change in medial temporal lobe subregions is associated with later memory decline in elderly controls. Using highresolution, T1-weighted magnetic resonance images acquired at baseline and six months follow-up, change in cortical thickness and subcortical volumes was measured in 142 healthy elderly subjects (aged 59-90 years) from the ADNI cohort. Regression analysis was used to identify whether change in fourteen subregions, selected a priori, was associated with declining performance on memory tests from baseline to two years follow-up. Percent thickness change in the right fusiform and inferior temporal cortices and expansion of the right inferior lateral ventricle were found to be significant predictors of subsequent decline on memory-specific neuropsychological measures. These results demonstrate that six-month regional neurodegeneration can be quantified in the healthy elderly and might help identify those at risk for subsequent cognitive decline.
\end{abstract}

\section{Keywords}

quantitative neuroimaging; aging; dementia; neurodegeneration; MRI; Alzheimer's Disease

\footnotetext{
(C) 2010 Elsevier Inc. All rights reserved.

"Corresponding author: James B. Brewer, M.D., Ph.D., Human Memory Laboratory, 8950 Villa La Jolla Drive C212, La Jolla, CA 92037, Tel: (858) 534-1237; Fax: (858) 534-1240, jbrewer@ucsd.edu.

Data used in the preparation of this article were obtained from the Alzheimer's Disease Neuroimaging Initiative (ADNI) database (www.loni.ucla.edu/ADNI). As such, the investigators within the ADNI contributed to the design and implementation of ADNI and/or provided data but did not participate in analysis or writing of this report. A complete listing of ADNI investigators is available at http://www.loni.ucla.edu/ADNI/Collaboration/ADNI_Manuscript_Citations.pdf.

Publisher's Disclaimer: This is a PDF file of an unedited manuscript that has been accepted for publication. As a service to our customers we are providing this early version of the manuscript. The manuscript will undergo copyediting, typesetting, and review of the resulting proof before it is published in its final citable form. Please note that during the production process errors may be discovered which could affect the content, and all legal disclaimers that apply to the journal pertain.
} 


\section{Introduction}

Loss of cognitive function during the transition from health to dementia can be linked to regional damage as pathological processes spread in the brain. Previous research suggests that the neurodegeneration occurring during this pathological spread can be quantified and used to predict further cognitive decline. For example, a quantification of neurodegeneration in patients with mild cognitive impairment (MCI) has been shown to predict progression to Alzheimer's disease (AD) (McEvoy et al., 2009). However, few studies have attempted to quantitatively assess regional neurodegeneration during the preclinical stage, before the onset of memory symptoms, and to see whether it is predictive of which individuals will subsequently undergo cognitive decline at a rate faster than that of normal aging. This has been challenging, in part, because healthy elderly subjects show minimal cognitive decline over the time course of most studies and therefore a large numbers of subjects are needed. By enrolling more than 200 normal controls in a three-year observational study, the Alzheimer's Disease Neuroimaging Initiative (ADNI) has made it possible to measure changes in cortical thickness and subregional brain volumes in the healthy elderly, thus enabling identification of those individuals who are in the earliest stages of neurodegeneration. As disease-modifying interventions become available, early detection of neurodegenerative illness becomes imperative, especially since the preclinical stage may be the period most amenable to disease-modifying therapy.

Longitudinal magnetic resonance imaging (MRI) can be used as a tool to aid early diagnosis of neurodegenerative illness by providing information about the rate of brain tissue loss, as evidenced by changes in cortical thickness and subcortical volumes. Further, the measurement of subregional change, coupled with knowledge about regional progression of various neurodegenerative diseases, may assist in differential diagnosis. Prior studies using longitudinal MRI for early detection of dementia have obtained measures of change through manual tracing (Jack et al., 1997), automated procedures for global volumetric change such as the boundary shift integral (Ridha et al., 2008), or methods based on statistical parametric mapping, such as voxel-based morphometry (Ashburner et al., 2003). The automated method in this paper uses nonlinear registration between baseline and follow-up scans to calculate volumetric change on a voxel-by-voxel basis. This is combined with image segmentation and cortical surface reconstruction to obtain average structural change in each subregion (Holland et al., 2009). The method is high-throughput and requires little manual input, making it applicable to large research studies and promising for extension to the clinical setting. Further, it has been shown to be sensitive to changes in the healthy elderly over one-year intervals. A recent study using this method in the ADNI elderly control cohort found that all identified subcortical and ventricular regions except the caudate nucleus and fourth ventricle changed significantly over one year (Fjell et al., 2009). It remains unclear whether these structural changes might be detectable over an even shorter time-period, how they relate to cognitive performance, and whether they are a harbinger of later cognitive decline.

The aim of this study was to investigate whether six-month, subregional change in the medial temporal region could be detected in elderly subjects with no diagnosis of dementia upon entry to the study and if so, whether it is predictive of subsequent cognitive decline on memoryspecific tasks. High-resolution, three-dimensional MRI scans were acquired six months apart and analyzed to obtain percentage change across fourteen cortical and subcortical regions. These fourteen regions for which longitudinal volumetric change measures were publicly available for download from the ADNI database on January 1,2010 were selected for inclusion in a regression analysis. In addition to regional temporal lobe measures, global measures of neurodegeneration were analyzed using change in cerebral and ventricular volumes.

Four memory-specific neuropsychological measures derived from the Logical Memory Test (from the Wechsler Memory Scale-Revised) and the Rey Auditory Verbal Learning Test 
(AVLT) were chosen as clinically relevant indicators of decline. Two-year changes in scores were used as distinct outcomes in separate regression analyses. Memory-specific tests were chosen as outcome measures because it is well recognized that memory impairment is an early symptom of Alzheimer's disease, and it has been proposed that tests specifically designed to measure memory functioning are sensitive to detecting early AD (Pike and Savage, 2008). Two of the neuropsychological measures were specifically chosen to assess episodic memory since impairment in this realm is considered a hallmark symptom of early AD (Sperling, et al., 2010). Further, performance on these specific tests has been previously associated with neurodegeneration in the medial temporal region (Apostolova et al., 2010).

Based on previous findings, the hypotheses were that six-month neurodegeneration could be detected in healthy elderly subjects, and that this neurodegeneration could be used to predict subsequent deterioration in memory performance as measured by neuropsychological testing.

\section{Methods}

\subsection{Alzheimer's Disease Neuroimaging Initiative}

Raw data used in this paper were obtained from the Alzheimer's Disease Neuroimaging Initiative (ADNI) public database (http://www.loni.ucla.edu/ADNI/). ADNI is a multi-site, five-year observational study of elderly individuals started in 2004 by the National Institute on Aging, the National Institute of Biomedical Imaging and Bioengineering, the Food and Drug Administration, private pharmaceutical companies and nonprofit organizations. Elderly controls, subjects with mild cognitive impairment (MCI) and AD subjects underwent longitudinal MRI scans and neuropsychological assessment at specified intervals for two to three years.

The Principal Investigator of this initiative is Michael W. Weiner, M.D. at the VA Medical Center and University of California, San Francisco. ADNI is the result of the efforts of many co-investigators from a broad range of academic institutions and private corporations, and subjects have been recruited from over 50 sites across the U.S. and Canada. For more information, see www.adni-info.org.

\subsection{Standard protocol approvals, enrolment and patient consents}

ADNI was conducted according to Good Clinical Practice guidelines, the Declaration of Helsinki, US 21CFR Part 50-Protection of Human Subjects, and Part 56-Institutional Review Boards, and pursuant to state and federal HIPAA regulations. Written informed consent for the study was obtained from all subjects and/or authorized representatives and study partners (http://www.ADNI-info.org).

\subsection{Subjects}

ADNI eligibility criteria are described at http://adni-info.org/TakingPartInADNI.aspx. In general, all enrolled subjects were between 55 and 90 (inclusive) years of age, had a study partner available to provide an independent evaluation of functioning, and spoke either English or Spanish. Control subjects were cognitively normal, based on an absence of significant impairment in cognitive functions or activities of daily living, and were in good general health with no depression or additional diseases expected to interfere with the study. They had baseline Mini Mental State Examination (MMSE) scores between 24 and 30 (inclusive), global Clinical Dementia Ratings (CDR) of 0 , and normal memory function determined by specific cutoffs on the Logical Memory Test (delayed paragraph recall) from the Wechsler Memory ScaleRevised. All control subjects were given the diagnosis of normal at baseline by physicians who were experienced neurologists or psychiatrists. This diagnosis was reviewed by physicians at 
each follow-up visit after 6 months, 12 months and 24 months to determine whether there was conversion to $\mathrm{MCI}$ or $\mathrm{AD}$.

In this study, data were included on all ADNI control subjects for whom the following criteria were satisfied by December 2009: baseline and six-month MR imaging data were available and passed a quality control review (described in Holland et al., 2009), and baseline and 24month neuropsychological data were available. Of the 229 ADNI control subjects, 142 were included and 87 were excluded. Demographics of the included subjects are described in Table 1. The differences between included and excluded subjects for mean age ( 75.88 vs 76.49 years, respectively), percent female subjects (.47 vs .49), mean education level (16.10 vs 15.94), percent carriers of the APOE $\varepsilon 4$ allele (.28 vs .24), and mean MMSE at baseline (29.22 vs. 28.94) did not meet statistical significance at the $\alpha=0.05$ level. However, the included subjects had significantly higher global cognition at baseline on mean Alzheimer's Disease Assessment Scale-Cognitive (ADAS-cog) (5.89 vs. 6.69, $\mathrm{p}=0.046)$.

\subsection{Neuropsychological assessment}

Two memory-specific neuropsychological tests administered at baseline and 24 months were used to determine cognitive decline: Logical Memory Test (Delayed Paragraph Recall) from the Wechsler Memory Scale-Revised and Rey Auditory Verbal Learning Test (AVLT). In the Logical Memory (LM) Test, which assesses episodic memory, subjects were read aloud a short story containing 25 units of information. The total number of units (maximum score $=25$ ) recalled immediately and after a 30-minute delay were recorded. Retention of the information was computed by dividing the delayed recall score by the immediate recall score. Two-year change in both the delayed recall score and the retention score were calculated as absolute change between baseline and 24 months, where cognitive decline was defined as a positive value.

The AVLT assesses multiple aspects of learning and memory. Subjects participated in five learning trials in which they were presented orally with the same 15 unrelated nouns at the rate of one word per second. The number of words freely recalled immediately after each trial was recorded for each of the 5 trials. After the first five trials, a second list of words was read, and the subjects were asked to freely recall as many words as possible immediately afterward (trial B). After a 20-minute delay, free recall of the original 15 words was elicited again (trial 6). Additionally, a yes/no recognition test containing the 15 original words interspersed with 15 new words was administered, and the numbers of true and false positive responses were recorded (delayed recognition). Two-year change in both the sum across all trials 1 through 6 plus B (maximum score $=105$ ) and the delayed recognition score (maximum score $=15$ ) were calculated as absolute change between baseline and 24 months, where cognitive decline was defined as a positive value.

\subsection{MR acquisition and analysis}

Two three-dimensional, T1-weighted volumes per subject per visit were downloaded from the public ADNI database (http://www.loni.ucla.edu/ADNI/). All image processing and analyses occurred at the Multimodal Imaging Laboratory at the University of California, San Diego. The methods are described in detail in Holland et al., 2009. Briefly, images were corrected for gradient nonlinearities (Jovicich et al., 2006). The two baseline images were rigid-body aligned, averaged to improve signal-to-noise ratio and resampled to isotropic 1-mm voxels. An automated segmentation procedure based on FeeSurfer software (Fischl, et al., 2002) and customized Matlab code was used to obtain volumetric segmentation. Cortical surface reconstruction yielded a measure of thickness at each vertex, and the surface was parceled into distinct regions of interest (ROIs). To obtain six-month change, follow-up images were fully affine-registered to the baseline images and corrected for intensity nonuniformity. Nonlinear 
registration was then used to align voxel centers in the baseline with the appropriate location in the follow-up scans, and a volume-change field was calculated. This field was averaged over each ROI to compute the percentage change from baseline. This method has been validated using models where amount of change was known and where noise was added to approximate that seen in human brain imaging of demented patients. In these studies, technical measurement error for the computed change in volume was within 0.2 percent of the structure volume. That is, for a $6000 \mathrm{~mm}^{3}$ hippocampal volume undergoing a change of 1 percent, the technical measurement error in estimating the $60 \mathrm{~mm}^{3}$ volumetric change would be $\pm 12 \mathrm{~mm}^{3}$ in an individual subject if voxels were each $1 \mathrm{~mm}$ isotropic (Holland, submitted).

\subsection{Statistical analysis}

All statistical analysis was performed using SPSS 15.0. To determine whether the population of subjects included in this study were representative of all ADNI control subjects, independent samples t-tests were used to compare age, sex, education, $A P O E \varepsilon 4$ carrier status, and baseline cognition (MMSE and ADAS-cog) with a significance level of $\alpha=0.05$.

To investigate whether six-month change in subregional brain volumes predicts two-year cognitive decline, regression analysis was used. Four different neuropsychological measures were treated as distinct dependent variables, and a separate regression analysis was done for each. The four outcome measures include two-year change in scores on LM delayed recall, LM retention, AVLT sum and AVLT delayed recognition. Six-month change in the whole brain, ventricles and twelve subregions of interest were selected to be independent variables in the regression analyses because these were the regions for which longitudinal data was available in the public ADNI database. The regions of interest included bilateral hippocampus, inferior lateral ventricle, middle temporal gyrus, inferior temporal gyrus, entorhinal cortex and fusiform gyrus. Risk factors for AD including age, sex, education and APOE $\varepsilon 4$ carrier status were also included in the regression analysis as independent variables.

Univariate analyses were first conducted to determine which independent variables should be included in a stepwise multivariate regression. All variables that met significance at $\mathrm{p}<.10$ in the univariate analyses were entered into the multivariate model. Residual analysis was conducted to evaluate the validity of regression assumptions. Additionally, independent variables that met significance $(\mathrm{p}<.01)$ in the multivariate model were tested for multicollinearity and undue influence. If a subject was found to have undue influence on the regression model, the subject's data was examined for abnormalities, the analysis was repeated after excluding the subjects with undue influence, and both results were reported.

To determine whether six-month neuroanatomical change provides additional predictive information beyond that which is obtained by assessing six-month change in cognition, the regression analysis was repeated using baseline ADAS-cog scores and six-month change in ADAS-cog, MMSE, AVLT sum and AVLT delayed recognition as independent variables. Sixmonth change in LM delayed recall and retention were not included because these measures were not assessed in the ADNI dataset at six months. Because six-month change on AVLT sum and AVLT delayed recognition are encompassed within two-year change on these same measures and are therefore not independent variables, the dependent variable in these analyses was change between the six-month and two-year visits rather than between the baseline and two-year visits.

A secondary set of regression analyses was also performed to identify how the multivariate models change when data from the seven subjects who converted to MCI over the course of two years is weighted twice as heavily as data from subjects who retained a diagnosis of normal. After assigning a weight of 2/149 (0.013423) to all data from subjects who converted to MCI 
and a weight of $1 / 149(0.006711)$ to all other subjects, the regression procedures detailed above were repeated.

Composite neuroanatomical change scores were calculated for each subject by converting each subject's change within the regional temporal lobe ROIs to standardized z-scores and then averaging the scores across these ROIs. Similarly, composite cognitive change scores were calculated for each subject by converting each subject's change on the neuropsychological measures to standardized z-scores and then averaging these scores across the four outcome measures.

\section{Results}

Of the 142 healthy elderly subjects included in this study, there were 67 females and 75 males, the mean age was 75 years (range 59 to 90), the mean education 16 years (range 6 to 20), and 40 were carriers of the APOE $\varepsilon 4$ allele. The mean MMSE at baseline was 29.22 (range 25 to 30 ) and the mean ADAS-cog at baseline was 5.89 (range 1 to 16.33).

On three of the four memory-specific neuropsychological assessments, subjects showed a slight mean improvement with repeat testing over the course of two years. The two-year mean change on LM delayed recall (maximum score 25) was an improvement of 0.64 points (range 10-point decline to 9-point improvement), the mean change on LM retention (maximum score 1) was a decline of 0.05 points (range 0.93 -point decline to 0.43 -point improvement), the mean change on AVLT sum (maximum score 105) was an improvement of 1.20 points (range 42point decline to 30-point improvement), and the mean change on AVLT delayed recognition (maximum score 15) was an improvement of 0.09 points (range 11-point decline to 10-point improvement).

There was significant evidence of medial temporal atrophy over a six-month period (Fig. 1). Although not all subjects had measurable atrophy, there was a mean loss of subcortical volume and cortical thickness in all regions of interest, with the exception of the inferior lateral ventricles, which expanded. There were mean decreases in the volumes of the left and right hippocampus (-1.02 and $-0.98 \%$, respectively) and mean decreases in the thicknesses of the left and right middle temporal gyri (both $-0.64 \%)$, left and right inferior temporal gyri (-0.55 and $-0.65 \%)$, left and right entorhinal cortices (-0.45 and $-0.43 \%)$, and left and right fusiform gyri $(-0.55$ and $-0.50 \%$, respectively). There were mean expansions in the volumes of the left and right inferior lateral ventricles ( 1.74 and $1.90 \%$, respectively). Additionally, there was a mean loss of volume in the whole brain $(-0.31 \%)$ and a mean gain in volume in all ventricles $(1.73 \%)$.

Univariate regression analysis revealed that six-month neurodegenerative change in several medial temporal regions significantly predicted two-year cognitive decline at a significance level $\alpha<.01$ (Table 2). The multivariate regression models designated three of these regions as the best predictors of cognitive decline when compared to other risk factors such as age, education and $A P O E \varepsilon 4$ carrier status. A decrease in the thickness of the right fusiform gyrus predicted decline on LM delayed recall $(\mathrm{p}=.001)$, with a $1 \%$ decrease in thickness corresponding to a $1.22 \pm 0.35$ point decline. Expansion of the right inferior lateral ventricle was a significant predictor of decline on LM retention $(\mathrm{p}=.002)$, with an expansion of $10 \%$ corresponding to a decline in the retention ratio by $0.09 \pm 0.03$. A decrease of thickness in the right inferior temporal gyrus predicted decline on AVLT sum $(\mathrm{p}=.004)$, with a $1 \%$ decrease in thickness corresponding to a $2.57 \pm .43$ point decline. Additionally, a decrease in whole brain volume predicted decline on AVLT delayed recognition ( $\mathrm{p}=.009)$ with a $1 \%$ decrease in volume corresponding to a $1.39 \pm .53$ point decline. Age, sex, education and APOE $\varepsilon 4$ carrier status were not significant predictors of cognitive decline. 
When baseline ADAS-cog scores and six-month change in ADAS-cog, and MMSE scores were entered into the regression model as independent variables, they failed to be significant predictors of two-year cognitive decline and the regression models did not change. Six-month change on AVLT sum did predict change on AVLT sum over the subsequent eighteen months $(\mathrm{p}=.000)$, and six-month change on AVLT delayed recognition predicted change on AVLT delayed recognition over the subsequent eighteen months ( $\mathrm{p}=.000)$. In addition, six-month change on AVLT sum predicted two-year change on LM delayed recall $(\mathrm{p}=.007)$.

Data from two subjects who converted to MCI during the two-year follow-up period were found to exert undue influence in the regression models for LM delayed recall and LM retention. When these subjects were removed from the regression analyses, neither the right fusiform nor the right inferior lateral ventricle were significant predictors at $\mathrm{p}<.01$, although the right fusiform remained significant at $\mathrm{p}<.05$. Change in the right fusiform predicted decline on LM delayed recall $(\mathrm{p}=.016)$, with a $1 \%$ decrease in thickness corresponding to a $0.92 \pm$ 0.38 point decline. Additionally, change in the left hippocampus became significant $(\mathrm{p}=.012)$ with a $1 \%$ decrease in thickness corresponding to a decline in the retention ratio by $0.39 \pm .15$.

During the two-year follow-up period, a total of seven subjects converted to MCI. The subjects who converted were not significantly different at baseline in age, education or MMSE than those who retained a normal diagnosis. However, the converters had a significantly higher frequency of the $A P O E \varepsilon 4$ allele $(0.71 \mathrm{vs} 0.26, \mathrm{p}=.049)$ and significantly more impaired ADAS-cog scores at baseline (mean 9.19 vs. $5.72, \mathrm{p}=.030$ ).

In a secondary regression analysis, data from the seven subjects who converted to MCI, including the two subjects mentioned above who exerted undue influence in the regression models, were weighted twice as heavily as data from all other subjects in order to create a prediction model biased toward identifying changes in the brain that predict conversion to MCI (Table 3). Change in the right fusiform became a more significant predictor ( $<$ <.001) of decline on $\mathrm{LM}$ delayed recall, with a $1 \%$ decrease in thickness corresponding to a $1.46 \pm .33$ point decline. Additionally, change in the right fusiform became a significant predictor $(\mathrm{p}=.005)$ of AVLT delayed recognition, with a $1 \%$ decrease in volume corresponding to a $.83 \pm .29$ point decline. Expansion of the right inferior lateral ventricle also became a more significant predictor $(\mathrm{p}<.001)$ of decline on LM retention, with a $10 \%$ increase corresponding to a decline in the ratio by $0.10 \pm 0.03$. No regions remained significant predictors of decline on AVLT sum at the $\alpha=.01$ level, but there was a trend toward change in the left entorhinal cortex predicting decline on AVLT sum $(\mathrm{p}=.017)$, with a $1 \%$ decrease in thickness corresponding to a $1.34 \pm .55$ point decline.

Among the nine subjects with the greatest composite neuroanatomical change (defined as being greater than one standard deviation relative to our sample), two converted to a diagnosis of $\mathrm{MCI}$, and five had cognitive change greater than the mean for the group. In no case did a subject above the 93rd percentile for composite neuroanatomical change fail to have greater than average decline on the composite memory measure. Further, in no case did a subject below the 32 nd percentile for composite neuroanatomical change convert to a diagnosis of MCI.

\section{Discussion}

This study provides evidence that loss of cortical thickness and subcortical volume can not only be detected and quantified over a six-month period in healthy elderly subjects, but can also be used to predict subsequent cognitive decline. Specifically, six-month neurodegeneration in certain medial temporal regions, including the right fusiform gyrus, right inferior lateral ventricle and right inferior temporal gyrus, predicted two-year decline on memory-specific neuropsychological measures in ADNI normal controls. These results 
suggest that longitudinal MRI may provide an early biomarker for cognitive decline in healthy elderly subjects. Further, the predictive information gained by longitudinal MRI cannot be obtained by assessing six-month change in global cognitive scores such as ADAS-cog or MMSE. It is less obvious that the usefulness of six-month MRI data in predicting subsequent decline on memory-specific measures exceeds that of six-month scores on these same memoryspecific tests. Six-month change on AVLT sum and AVLT delayed recognition were found to be significant predictors of change on these same tests over the subsequent eighteen months. However, they did not predict change on one another and only AVLT sum predicted two-year change on LM delayed recall. At a minimum, the information provided by six-month imaging data enhances information gained by extensive neuropsychological testing. Further, imaging measures have certain advantages over neuropsychological tests in that they are not influenced by volition or attention.

This study is the first to detect and quantify six-month change across multiple medial temporal regions in healthy elderly subjects and to relate these changes to later cognitive decline with a relatively short follow-up. Prior studies have examined a limited number of structures using volumes obtained through manual tracing (Jack et al., 1997) or qualitative ratings (Scheltens et al., 1992). The current procedure is high-throughput and requires little manual input, thus making it applicable to the clinical setting or large research studies.

Other studies examining neurodegeneration in healthy elderly subjects have focused on either baseline volumes or change over longer time periods, at least one year in duration. It is likely that additional information is gained by including baseline volumes in a predictive model (Adak et al, 2004), but the purpose of this study was to specifically examine change over a sixmonth period. Baseline volumes can be interpreted as the sum of all changes occurring prior to the six-month period of interest. These results show that regardless of baseline volumes, a six-month follow-up is sufficient to identify neurodegenerative changes. Because six months is the shortest follow-up period in the ADNI dataset, it remains to be seen whether neurodegenerative changes could be detected in even shorter time periods, such as three months. Further studies are needed to compare the quality of predictive information gained by assessing change over different time periods.

It is unclear whether the neurodegeneration detected in this study is entirely explained by general aging or whether early pathological processes occurring in some subjects drive the prediction models. It is well established that there is a gradual loss of brain volume with normal aging (Scahill et al., 2003), and there is not sufficient follow-up in the ADNI dataset to determine if these subjects will go on to develop AD. However, the pattern of neurodegeneration seen in the subjects whose subregional brain volumes change the most in six months is consistent with what is known about the regional progression of pathology in $\mathrm{AD}$, which starts in medial temporal lobe structures and spreads laterally through the neocortex (Braak and Braak, 1991). Although prior studies have focused on early changes in the hippocampus and entorhinal cortex as being predictive of AD (Devanand, et al. 2007; Hampel, et al., 2008), the fusiform gyrus, inferior lateral ventricles and inferior temporal gyrus lie along the known path of pathological progression and would be expected to change in disease as well. Further, the findings of this study are consistent with prior studies in MCI and AD that have found significant volume changes in the fusiform gyrus (Holland et al., 2009; McDonald, 2009), inferior temporal gyrus (Holland et al., 2009; McDonald, 2009) and inferior lateral ventricles (Kovacevic et al., 2009). Thus, it is reasonable that these three regions were the best predictors of cognitive decline in the regression models.

Additionally, there is information about the seven subjects who did convert to a diagnosis of MCI over the two-year follow-up. Although not enough subjects converted to MCI to provide sufficient statistical power, a qualitative analysis of their imaging data reveals that the six- 
month change seen in their brains is highly consistent with what is expected in the early forms of AD (Fig. 2). These seven subjects, in whom a clinically relevant change was noted, are clearly of particular interest. The secondary regression analysis was used to examine how the regression models would change when their data was weighted more heavily, thus influencing the regression models toward the specific changes that occurred in subjects known to be converters. In doing so, subregional change in the fusiform gyrus and inferior lateral ventricle became stronger predictors of cognitive decline. Again, these regions are consistent with the known path of pathologic progression in $\mathrm{AD}$, and it is therefore likely that accelerated change in these regions suggests pathology rather than general aging although longer follow-up and pathological confirmation would be required to confirm this.

When further examining the seven subjects who converted to MCI, it appears that $A P O E$ genotype is meaningful. Five of the seven converters were carriers of the APOE $\varepsilon 4$ allele, compared to 35 carriers of the allele among the 135 subjects who did not convert to MCI. This suggests that $A P O E$ genotype may assist in knowing whether neurodegenerative change detected by quantitative neuroimaging is clinically relevant and likely to result in a change in diagnosis from normal cognition to MCI.

Although the pattern of atrophy observed in some subjects in this study is consistent with expected changes in AD, the hemispheric asymmetry is unexpected. Namely, these results suggest that atrophy in right hemisphere regions is more strongly predictive of later cognitive decline than is atrophy in the left hemisphere. Many prior studies have reported greater involvement of the left hemisphere than the right hemisphere in AD (Karas et al., 2003; Baron et al., 2001) and MCI (Seo et al., 2007) although one study found greater atrophy in the right medial temporal region than in the left in MCI (Karas et al., 2004), and another study using the ADNI dataset reported greater thinning in the right hippocampal and entorhinal cortices in patients with MCI (Fennema-Notestine et al., 2009). It is important to note that the results of this study do not suggest that greater atrophy occurs in right hemispheric regions. In fact, mean percent changes in volume were not statistically different between the right and left hemispheres for any regions examined. However, the results do suggest that right hemispheric atrophy is more predictive of later cognitive decline. It is unclear why such a laterality exists, and this is an issue that merits further study.

Finally, despite the potential for future application of these procedures in the clinical setting, it should be mentioned that such use would require additional investigation into their power to detect such subtle changes in the individual subject, as well as overcoming a host of other practical issues that have impeded the translation of volumetry into the clinical setting (Brewer et al, 2009). Further, the ADNI dataset does not accurately represent the population at large, which limits generalizability of the findings. In particular, the normal subjects included in this study had a high average level of education ( 16.12 years) compared to the general population. This warrants further research because other investigators have found that low education levels are associated with an increased risk of dementia (Chen et al., 2009). Thus, one might expect more neurodegeneration and cognitive decline in a sample more representative of the general population.

\section{Conclusion}

The present findings suggest that longitudinal MRI shows promise for identifying rapid atrophy in otherwise healthy subjects. It is too early to tell whether the atrophy identified in this population represents significant disease that should be targeted for therapy, especially since histological underpinnings might vary. Nevertheless, the results suggest that such MR neuroimaging techniques might be used to supplement existing research and diagnostic techniques to evaluate risk of memory decline. For example, this subregional approach using 
longitudinal MRI may supplement measures of global atrophy currently used in clinical trials to assess whether or not a therapeutic agent slows brain atrophy and associated decline in memory. Neuroimaging may even offer an advantage over cognitive testing in clinical trials targeting the earliest disease stages by allowing earlier detection of a drug's effect in the prodromal phase, when an effect on atrophy would appear to be demonstrable prior to an effect on cognition. Thus, these techniques may have significant future implications for both diagnosis and prognosis, even among the healthy elderly.

\section{Acknowledgments}

Data collection and sharing for this project was funded by the Alzheimer's Disease Neuroimaging Initiative (ADNI) (National Institutes of Health Grant U01 AG024904). ADNI is funded by the National Institute on Aging, the National Institute of Biomedical Imaging and Bioengineering, and through generous contributions from the following: Abbott, AstraZeneca AB, Bayer Schering Pharma AG, Bristol-Myers Squibb, Eisai Global Clinical Development, Elan Corporation, Genentech, GE Healthcare, GlaxoSmithKline, Innogenetics, Johnson and Johnson, Eli Lilly and Co., Medpace, Inc., Merck and Co., Inc., Novartis AG, Pfizer Inc, F. Hoffman-La Roche, Schering-Plough, Synarc, Inc., and Wyeth, as well as non-profit partners the Alzheimer's Association and Alzheimer's Drug Discovery Foundation, with participation from the U.S. Food and Drug Administration. Private sector contributions to ADNI are facilitated by the Foundation for the National Institutes of Health (www.fnih.org). The grantee organization is the Northern California Institute for Research and Education, and the study is coordinated by the Alzheimer's Disease Cooperative Study at the University of California, San Diego. ADNI data are disseminated by the Laboratory for Neuro Imaging at the University of California, Los Angeles. This research was also supported by NIH grants P30 AG010129, K01 AG030514, and the Dana Foundation.

J.B.B. is supported by NINDS K23NS050305; E.A.M. is supported in part by NIGMS Training Grant GM007198.

A.M.D. receives funding to his laboratory from General Electric Medical Systems as part of a Master Research Agreement with UCSD; and is a founder of, holds equity in, and serves on the scientific advisory board for CorTechs Labs, Inc. The terms of this arrangement have been reviewed and approved by UCSD in accordance with its conflict of interest policy.

\section{References}

Adak S, Illouz K, Gorman W, Tandon R, Zimmerman EA, Guariglia R, Moore MM, Kaye JA. Predicting the rate of cognitive decline in aging and early Alzheimer disease. Neurology 2004;63(1):108-14. [PubMed: 15249619]

Apostolova LG, Morra JH, Green AE, Hwang KS, Avedissian C, Woo E, Cummings JL, Toga AW, Jack CR Jr, Weiner MW, Thompson PM. Alzheimer's Disease Neuroimaging Initiative. Automated 3D mapping of baseline and 12-month associations between three verbal memory measures and hippocampal atrophy in 490 ADNI subjects. Neuroimage 2010;51(1):488-99. [PubMed: 20083211]

Ashburner J, Csernansky JG, Davatzikos C, Fox NC, Frisoni GB, Thompson PM. Computer-assisted imaging to assess brain structure in healthy and diseased brains. Lancet Neurol 2003;2(2):79-88. [PubMed: 12849264]

Baron JC, Chetelat G, Desgranges B, Perchey G, Landeau B, de la Sayette V, Eustache F. In vivo mapping of gray matter loss with voxel-based morphometry in mild Alzheimer's disease. Neuroimage 2001;14 (2):298-309. [PubMed: 11467904]

Braak H, Braak E. Neuropathological staging of Alzheimer-related changes. Acta Neuropathol 1991;82:239-259. [PubMed: 1759558]

Brewer JB. Fully-automated volumetric MRI with normative ranges: translation to clinical practice. Behav Neurol 2009;21(1):21-8. [PubMed: 19847042]

Chen JH, Lin KP, Chen YC. Risk factors for dementia. J Formos Med Assoc 2009;108(10):754-64. [PubMed: 19864195]

Devanand DP, Pradhaban G, Liu X, Khandji A, De Santi S, Segal S, Rusinek H, Pelton GH, Honig LS, Mayeux R, Stern Y, Tabert MH, de Leon MJ. Hippocampal and entorhinal atrophy in mild cognitive impairment: prediction of Alzheimer disease. Neurology 2007;68(11):828-36. [PubMed: 17353470]

Fennema-Notestine C, Hagler DJ Jr, McEvoy LK, Fleisher AS, Wu EH, Karow DS, Dale AM. Alzheimer's Disease Neuroimaging Initiative. Structural MRI biomarkers for preclinical and mild Alzheimer's disease. Hum Brain Mapp 2009;30(10):3238-53. [PubMed: 19277975] 
Fischl B, Salat DH, Busa E, Albert M, Dieterich M, Haselgrove C, van der Kouwe A, Killiany R, Kennedy D, Klaveness S, Montillo A, Makris N, Rosen B, Dale AM. Whole brain segmentation: automated labeling of neuroanatomical structures in the human brain. Neuron 2002;33(3):341-55. [PubMed: 11832223]

Fjell AM, Walhovd KB, Fennema-Notestine C, McEvoy LK, Hagler DJ, Holland D, Brewer JB, Dale AM. One-year brain atrophy evident in healthy aging. J Neurosci 2009;29(48):15223-15231. [PubMed: 19955375]

Hampel H, Burger K, Teipel SJ, Bokde AL, Zetterberg H, Blennow K. Core candidate neurochemical and imaging biomarkers of Alzheimer's disease. Alzheimers Dement 2008;4(1):38-48. [PubMed: 18631949]

Holland D, Brewer JB, Hagler DJ, Fennema-Notestine C, Dale AM. and the Alzheimer's Disease Neuroimaging Initiative. Subregional neuroanatomical change as a biomarker for Alzheimer's disease. Proc Natl Acad Sci USA 2009;106(49):20954-20959. [PubMed: 19996185]

Jack CR Jr, Petersen RC, Xu YC, Waring SC, O’Brien PC, Tangalos EG, Smith GE, Ivnik RJ, Kokmen E. Medial temporal atrophy on MRI in normal aging and very mild Alzheimer's disease. Neurology 1997;49(3):786-794. [PubMed: 9305341]

Jovicich J, Czanner S, Greve D, Haley E, van der Kouwe A, Gollub R, Kennedy D, Schmitt F, Brown G, Macfall J, Fischl B, Dale A. Reliability in multi-site structural MRI studies: effects of gradient non-linearity correction on phantom and human data. Neuroimage 2006;30(2):436-43. [PubMed: 16300968]

Karas GB, Scheltens P, Rombouts SARB, Visser PJ, van Schijndel RA, Fox NC, Barkhof F. Global and local gray matter loss in mild cognitive impairment and Alzheimer's disease. Neuroimage 2004;23 (2):708-716. [PubMed: 15488420]

Karas GB, Burton EJ, Roumbouts SA, van Schijndel RA, O'Brien JT, Scheltens P, McKeith IG, Williams D, Ballard C, Barkhof F. A comprehensive study of gray matter loss in patients with Alzheimer's disease using optimized voxel-based morphometry. Neuroimage 2003;18(4):895-907. [PubMed: 12725765]

Kovacevic S, Rafii MS, Brewer JB. Alzheimer's Disease Neuroimaging Initiative. High-throughput, fully-automated volumetry for prediction of MMSE and CDR decline in mild cognitive impairment. Alzheimer Dis Assoc Disord 2009;23(2):139-145. [PubMed: 19474571]

McDonald CR, McEvoy LK, Gharapetian L, Fennema-Notestine C, Hagler DJ Jr, Holland D, Koyama A, Brewer JB, Dale AM. and the Alzheimer's Disease Neuroimaging Initiative. Regional rates of neocortical atrophy from normal aging to early Alzheimer disease. Neurology 2009;73(6):457-65. [PubMed: 19667321]

McEvoy LK, Fennema-Notestine C, Roddey JC, Hagler DJ Jr, Holland D, Karow DS, Pung CJ, Brewer JB, Dale AM. and for the Alzheimer's Disease Neuroimaging Initiative. Alzheimer Disease: Quantitative structural neuroimaging for detection and prediction of clinical and structural changes in Mild Cognitive Impairment. Radiology 2009;251(1):195-205. [PubMed: 19201945]

Pike KE, Savage G. Memory profiling in mild cognitive impairment: can we determine risk for Alzheimer's disease? J Neuropsychol 2008;2(Pt 2):361-72. [PubMed: 19824171]

Ridha BH, Anderson VM, Barnes J, Boyes RG, Price SL, Rossor MN, Whitwell JL, Jenkins L, Black RS, Grundman M, Fox NC. Volumetric MRI and cognitive measures in Alzheimer disease: comparison of markers of progression. J Neurol 2008;255(4):567-74. [PubMed: 18274807]

Scahill RI, Frost C, Jenkins R, Whitwell JL, Rossor MN, Fox NC. A longitudinal study of brain volume changes in normal aging using serial registered magnetic resonance imaging. Arch Neurol 2003;60 (7):989-994. [PubMed: 12873856]

Scheltens P, Leys D, Barkhof F, Huglo D, Weinstein HC, Vermersch P, Kuiper M, Steinling M, Wolters EC, Valk J. Atrophy of medial temporal lobes on MRI in "probable" Alzheimer's disease and normal ageing: diagnostic value and neuropsychological correlates. J Neurosurg Psychiatry 1992;55(10): 967-72.

Seo SW, Im K, Lee JM, Kim YH, Kim ST, Kim SY, Yang DW, Kim SI, Cho YS, Na DL. Cortical thickness in single- versus multiple-domain amnestic mild cognitive impairment. Neuroimage 2007;36(2):289-297. [PubMed: 17459730] 
Sperling RA, Dickerson BC, Pihlajamaki M, Vannini P, LaViolette PS, Vitolo OV, Hedden T, Becker JA, Rentz DM, Selkoe DJ, Johnson KA. Functional alterations in memory networks in early Alzheimer's disease. Neuromolecular Med 2010;12(1):27-43. [PubMed: 20069392] 

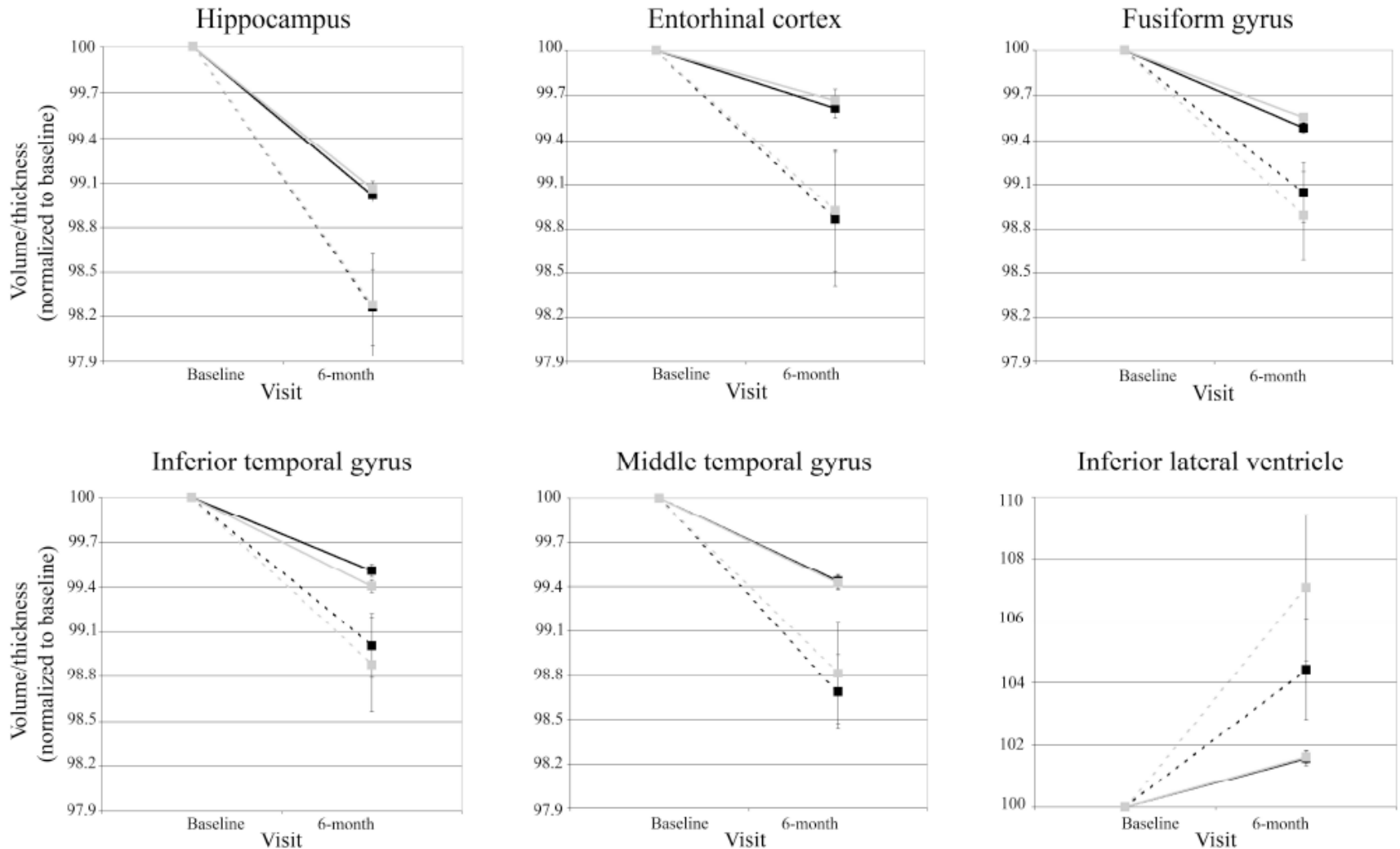

Middle temporal gyrus

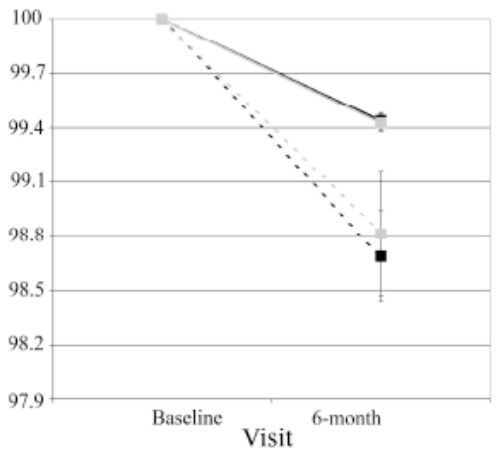

Inferior lateral ventricle

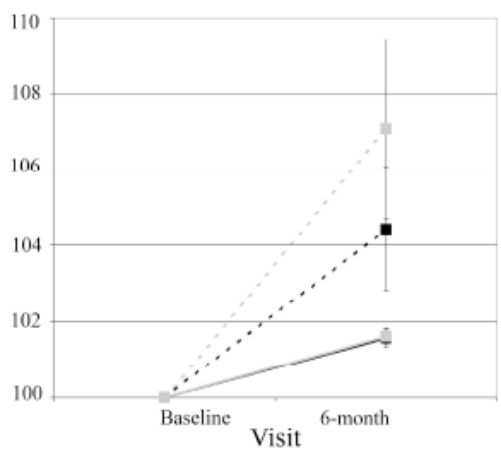

$\rightarrow-$ L hemisphere, non-converters $\quad \longrightarrow$ R hemisphere, non-converters $\quad \cdots \cdots$ L hemisphere, converters

R hemisphere, converters

Figure 1.

Neuroanatomical change between baseline and 6-month follow-up visits across medial temporal lobe (MTL) regions. All volumes were normalized to baseline. Data points represent mean volumes for all subjects who retained a diagnosis of cognitively normal over the twoyear follow-up (solid lines, $n=135$ ) and for those subjects who converted to mild cognitive impairment (MCI) during the follow-up (dashed lines, $n=7)$. Error bars represent standard error of the mean. A mean loss in volume was seen in all medial temporal lobe regions, with the exception of the inferior lateral ventricle, in which there was mean expansion. 

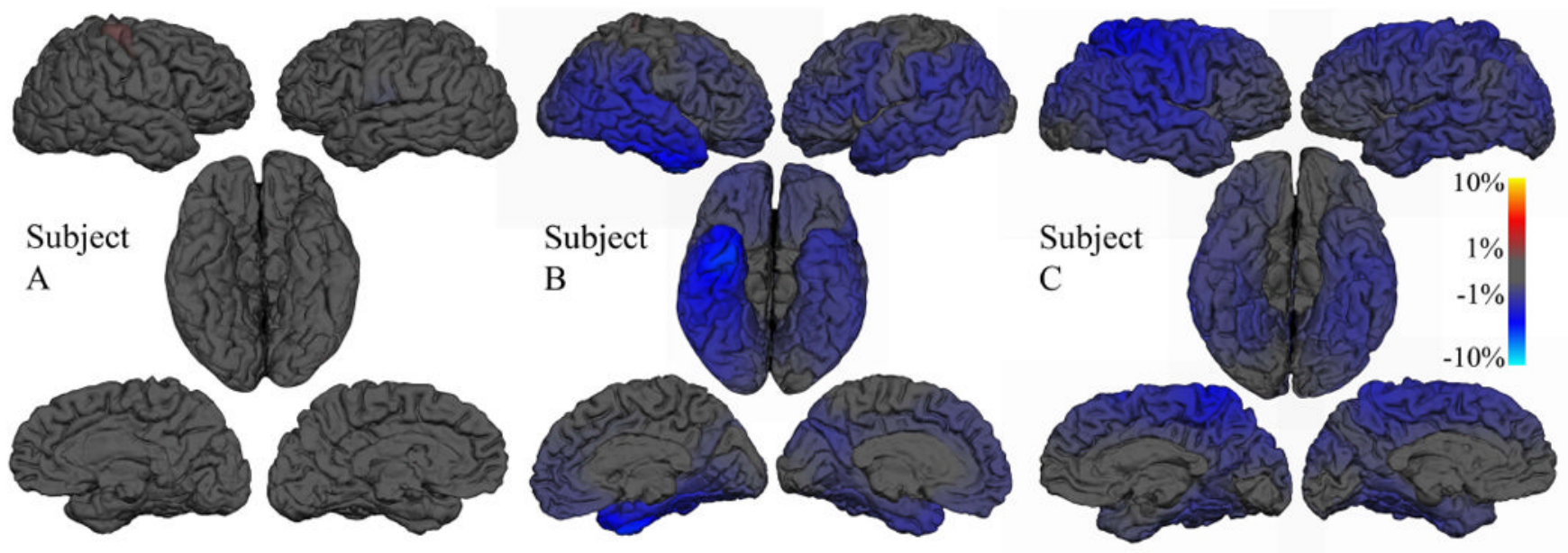

Figure 2.

Six-month neuroanatomical change overlaid on baseline brain images for three sample subjects using a heat-scale color mapping where blue represents shrinkage and red represents expansion. Subject A was a 77 year-old female who retained a diagnosis of cognitively normal throughout the two-year follow-up period. Subject B was a 77 year-old female who converted to a diagnosis of mild cognitive impairment (MCI) at the six-month follow-up. Subject $\mathrm{C}$ was a 79 year-old male who converted to a diagnosis of MCI at the 24-month follow-up. 


\section{Table 1}

Subject baseline characteristics

\begin{tabular}{|c|c|c|}
\hline & All $(\mathrm{n}=142)^{a c}$ & Conv. $(\mathrm{n}=7)^{b c}$ \\
\hline Age (years) & $75.88 \pm 4.86$ & $74.81 \pm 5.82$ \\
\hline Percent female subjects & $47 \%$ & $29 \%$ \\
\hline Education (years) & $16.12 \pm 2.73$ & $16.57 \pm 1.81$ \\
\hline Percent carriers of $A P O E \varepsilon 4$ & $28 \% *$ & $71 \% *$ \\
\hline Baseline MMSE & $29.22 \pm .92$ & $28.57 \pm .79$ \\
\hline Baseline ADAS-cog & $5.89 \pm 2.85^{*}$ & $9.19 \pm 3.26^{*}$ \\
\hline
\end{tabular}

${ }^{a}$ Contains information about all 142 subjects whose data was included in the analysis.

${ }^{b}$ Contains information about only those 7 subjects who converted to a diagnosis of mild cognitive impairment (MCI) during the two-year follow-up.

${ }^{c}$ Data are given as mean \pm SD unless otherwise specified as a percent.

The subjects who converted to $\mathrm{MCI}$ had a significantly higher frequency of the APOE $\varepsilon 4$ allele and significantly more impaired ADAS-cog scores at baseline $(\mathrm{p}<0.05)$. 
Table 2

Variables of significance in univariate and multivariate regression models

\begin{tabular}{|c|c|c|}
\hline & $\begin{array}{l}\underline{\text { Univariate }}^{b} \\
\mathrm{t}(\mathrm{p}<0.1)\end{array}$ & $\begin{array}{l}\underline{\text { Multivariate }}^{a b} \\
\mathrm{t}(\mathrm{p}<0.01)\end{array}$ \\
\hline \multicolumn{3}{|c|}{ Outcome: Logical Memory Delayed Recall } \\
\hline Right fusiform & $-3.190(.002)$ & $-3.453(.001)$ \\
\hline Left hippocampus & $-2.954(.004)$ & \\
\hline Right inferior lateral ventricle & $2.791(.006)$ & \\
\hline Right inferior temporal gyrus & $-2.490(.014)$ & \\
\hline Total cerebral & $-2.461(.015)$ & \\
\hline Right hippocampus & $-2.172(.032)$ & \\
\hline Left fusiform & $-2.058(.041)$ & \\
\hline Right entorhinal cortex & $-2.007(.047)$ & \\
\hline Left inferior lateral ventricle & $1.742(.084)$ & \\
\hline \multicolumn{3}{|c|}{ Outcome: Logical Memory Retention } \\
\hline Right inferior lateral ventricle & $3.066(.003)$ & $3.105(.002)$ \\
\hline Left hippocampus & $-2.536(.012)$ & \\
\hline Left inferior lateral ventricle & $2.424(.017)$ & \\
\hline Right fusiform & $-1.918(.057)$ & \\
\hline All ventricles & $1.876(.063)$ & \\
\hline \multicolumn{3}{|c|}{ Outcome: AVLT Sum } \\
\hline Right inferior temporal gyrus & $-2.935(.004)$ & $-2.935(.004)$ \\
\hline Left entorhinal cortex & $-2.605(.010)$ & \\
\hline Left hippocampus & $-2.244(.026)$ & \\
\hline Education & $-2.160(.033)$ & \\
\hline Right entorhinal cortex & $-1.851(.066)$ & \\
\hline
\end{tabular}

Outcome: AVLT Delayed Recognition

\begin{tabular}{l|l|l}
\hline Total cerebral & $\mathbf{- 2 . 7 6 5}(.006)$ & $\mathbf{- 2 . 6 3 9 ( . 0 0 9 )}$ \\
Right fusiform & $\mathbf{- 2 . 7 3 2 ( . 0 0 7 )}$ & \\
Right inferior temporal & $-2.113(.036)$ & \\
APOE & $2.043(.043)$ & \\
Age & $2.020(.045)$ & \\
Education & $-1.827(.070)$ & \\
Left hippocampus & $-1.687(.094)$ & \\
Left entorhinal cortex & $-1.673(.097)$ & \\
\hline
\end{tabular}

${ }^{a}$ All variables that met significance at $\mathrm{p}<0.1$ in the univariate analysis were included in the multivariate model.

$b$ Data is reported as t-value ( $\mathrm{p}$-value). Variables that met significance at $\mathrm{p}<0.01$ are highlighted in bold for both the univariate and multivariate analyses. 
Table 3

Variables of significance in regression analysis after weighting data from subjects who converted to $\mathrm{MCI}^{a}$

\begin{tabular}{|c|c|c|}
\hline & $\begin{array}{l}\underline{\text { Univariate }}^{c} \\
\mathrm{t}(\mathrm{p}<0.1)\end{array}$ & $\begin{array}{l}\underline{\text { Multivariate }}^{b c} \\
\mathrm{t}(\mathrm{p}<0.01)\end{array}$ \\
\hline \multicolumn{3}{|c|}{ Outcome: Logical Memory Delayed Recall } \\
\hline Right fusiform & $-4.118(\mathrm{p}<.000)$ & $-4.371(\mathrm{p}<.000)$ \\
\hline Right inferior lateral ventricle & $4.013(\mathrm{p}<.000)$ & \\
\hline Left hippocampus & $-3.708(p<.000)$ & \\
\hline Right inferior temporal gyrus & $-3.215(.002)$ & \\
\hline Total cerebral & $-3.150(.002)$ & \\
\hline Right hippocampus & $-3.048(.003)$ & \\
\hline Left fusiform & $-2.750(.007)$ & \\
\hline Left inferior lateral ventricle & $2.643(.009)$ & \\
\hline Right entorhinal cortex & $-2.625(.010)$ & \\
\hline Right middle temporal gyrus & $-2.424(.017)$ & \\
\hline All ventricles & $2.399(.018)$ & \\
\hline Left inferior temporal gyrus & $-1.925(.056)$ & \\
\hline Age & $1.906(.059)$ & \\
\hline \multicolumn{3}{|c|}{ Outcome: Logical Memory Retention } \\
\hline Right inferior lateral ventricle & $3.547(.001)$ & $3.577(\mathrm{p}<.000)$ \\
\hline Left hippocampus & $-2.889(.004)$ & \\
\hline Left inferior lateral ventricle & $2.704(.008)$ & \\
\hline Right fusiform & $-2.519(.013)$ & \\
\hline All ventricles & $2.444(.016)$ & \\
\hline Total cerebral & $-1.995(.048)$ & \\
\hline Right hippocampus & $-1.868(.064)$ & \\
\hline Left fusiform & $-1.813(.072)$ & \\
\hline Right middle temporal gyrus & $-1.681(.095)$ & \\
\hline \multicolumn{3}{|c|}{ Outcome: AVLT Sum } \\
\hline Right inferior temporal gyrus & $-3.034(.003)$ & \\
\hline Left entorhinal cortex & $-2.922(.004)$ & \\
\hline Left hippocampus & $-2.531(.013)$ & \\
\hline Right entorhinal cortex & $-2.086(.039)$ & \\
\hline Education & $-2.042(.043)$ & \\
\hline Right hippocampus & $-1.989(.049)$ & \\
\hline Right middle temporal gyrus & $-1.827(.070)$ & \\
\hline Right fusiform & $-1.779(.077)$ & \\
\hline
\end{tabular}




\begin{tabular}{|l|l|l|}
\hline & $\begin{array}{l}{\underline{\text { Univariate }^{c}}}^{\boldsymbol{t}(\mathbf{p}<\mathbf{0 . 1})} \\
\text { Right fusiform }\end{array}$ & ${\underline{\text { Multivariate }^{\boldsymbol{b}}}}^{\mathbf{t}(\mathbf{p}<\mathbf{0 . 0 1})}$ \\
Total cerebral & $\mathbf{- 3 . 1 3 3 ( . 0 0 2 )}$ & $\mathbf{- 2 . 8 4 1 ( . 0 0 5 )}$ \\
APOE & $\mathbf{- 3 . 0 0 5 ( . 0 0 3 )}$ & \\
Right inferior lateral ventricle & $\mathbf{2 . 7 4 1 ( . 0 0 7 )}$ & \\
Right inferior temporal gyrus & $-2.331(.021)$ & \\
Age & $2.097(.038)$ & \\
Left hippocampus & $-2.079(.039)$ & \\
Left entorhinal cortex & $-2.066(.041)$ & \\
Left fusiform & $-1.981(.050)$ & \\
Left inferior lateral ventricle & $1.777(.078)$ & \\
Right entorhinal cortex & $-1.697(.092)$ & \\
\hline
\end{tabular}

${ }^{a}$ Subjects who converted to a diagnosis of mild cognitive impairment (MCI) during the two-year follow-up were weighted twice as heavily as all other subjects in this regression analysis.

${ }^{b}$ All variables that met significance at $\mathrm{p}<0.1$ in the univariate analysis were included in the multivariate model.

${ }^{c}$ Data is reported as t-value (p-value). Variables that met significance at $\mathrm{p}<0.01$ are highlighted in bold for both the univariate and multivariate analyses. 